Astrophysics and Space Science manuscript No.

(will be inserted by the editor)

Gianfranco Bertone

\title{
Dark Matter: the Connection with Gamma-Ray Astrophysics
}

\author{
Discovering Dark Matter particles with astrophysical observations
}

Received: date / Accepted: date

\begin{abstract}
We review the status of indirect Dark Matter searches, focusing in particular on the connection with gamma-ray Astrophysics, and on the prospects for detection with the upcoming space telescope GLAST and Air Cherenkov Telescopes such as CANGAROO, HESS, MAGIC and VERITAS. After a brief introduction where we review the fundamental motivations for indirect searches, we tackle the question of whether it is possible to obtain strong enough evidence from astrophysical observations, to claim discovery of Dark Matter particles. To this purpose, we discuss some recent conflicting claims that have generated some confusion in the field, and present new strategies that may provide the , long-awaited smoking-gun for Dark Matter.
\end{abstract}

\section{Introduction}

Dark Matter (DM) is one of the pillars of Modern Cosmology, and cosmological observations provide the most robust (if not the only) evidence for physics beyond the Standard Model. The discovery of Dark Matter particles may thus represent at the same time the discovery of new physics, and the identification of one of the most fundamental consituents of the Universe (for reviews see e.g. Refs. 1,2]. Of course the best case scenario is that DM particles are found at accelerators, in which case astrophysical observations can be used to prove that the discovered particles have the appropriate cosmological abundance, and to further constrain their properties 3 .

But what if DM particles are not observed at accelerators, what if it takes many years to obtain conclusive answers from, say, the analysis of events at the Large Hadron Collider (LHC)? Is it possible to obtain conclusive enough evidence from astrophysical observations only, to actually claim "discovery" of DM particles?

DM searches can be broadly divided in three different categories. First, one could search for new particles at

INFN, Sezione di Padova, Via Marzolo 8, Padova I-35131, Italy accelerators. Much attention has in fact been devoted to the prospects for detecting new physics, and in particular signals of Supersymmetry (SUSY) with accelerators, such as Tevatron and the LHC. If new particles are discovered, it might be even possible, starting from the mass spectrum and particle properties of the new theory, to obtain a tentative estimate of the relic density of the new particles, thus allowing a tentative identification of the DM particle (see e.g. Refs. 4,5] and references therein).

The prospects for discovery at accelerators have been worked out also for an alternative candidate, i.e. the lightest Kaluza-Klein particle (LKP) 6] , in theories with Universal Extra Dimensions (UED) [7]. It might be possible already at LHC to discriminate between SUSY and UED, thus to obtain a first hint on the nature of DM 8 . Alternatively, one might search for DM particles directly, i.e. my measuring the energy recoil of a nucleus hit by a DM particle streaming through the Earth. More than 20 direct DM detection experiments are either now operating or are currently in development. We refer the interested reader to Refs. [1,2] for more details.

Finally, DM can be searched for indirectly, i.e. through the detection of its annihilation products such as gammarays, neutrinos and anti-matter (e.g. Ref. 2 and references therein). Although indirect searches are inevitably affected by large astrophysical uncertainties, it is nonetheless possible to obtain useful constraints on the properties of DM, and possibly, as we argue below, to obtain strong enough evidence to claim discovery.

The paper is organized as follows: we tackle some fundamental questions about the motivations for indirect DM searches in Sec. 2] whose title could be "Everything you always wanted to know about DM and never dared to ask". The experienced reader can skip this section and move directly to Sec. 3 where we provide a critical discussion of some recent conflicting claims of

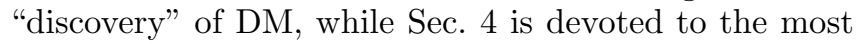
recently proposed strategies that could eventually provide the smoking-gun for DM annihilations. 


\section{Gamma ray - Dark Matter connection: FAQ}

The introductory material contained in this section is aimed at non-experts, and reviews the fundamental motivations for indirect searches. The experienced reader can skip this section and move directly to the next one, where we present an update on indirect searches.

As mentioned in the introduction, a number of good reviews on DM candidates and searches has recently appeared in literature. In the limited space available here, we will not attempt to provide an exhaustive review of the (many!) candidates proposed in literature, nor a description of the various detection strategies proposed over the last two decades. Instead, it is probably more appropriate to take a step back, and tackle here some fundamental questions, frequently asked at conferences and workshops by non-experts, e.g.: if DM is dark by definition, why should one expect any indirect signature, such as emission of gamma-rays, or neutrinos? Why most studies focus on annihilation rather than, say, decay of DM particles? Why so many experiments exploring the $\mathrm{GeV}-\mathrm{TeV}$ energy range, what motivates this mass scale? Finally, how natural are these scenarios? As we shall see, for some of these questions there is a precise answer, while for others the answer can only be tentative, reflecting our ignorance on what DM really is.

Why annihilations? DM is dark in the sense that there is no apparent electromagnetic emission associated with it. So why should one expect to detect any emission such as gamma-rays and neutrinos from DM? Why every indirect DM search focuses on DM annihilation rather than, say, decay, or interactions with ordinary matter?

To answer this question, we start from the standard DM paradigm, commonly adopted by cosmologists, where DM is made of some new particles, thermally produced in the Early Universe, kept in thermal and chemical equlibrium in the early Universe through pair annihilation into Standard Model particles, and Standard model processes leading to the production of DM particle pairs. The density of DM particles $n(t)$ is governed by the Boltzmann equation 9]

$$
\frac{\mathrm{d} n}{\mathrm{~d} t}+3 H n=-<\sigma v>\left(n^{2}-n_{e q}^{2}\right)
$$

where $\mathrm{H}$ is the Hubble parameter, $\langle\sigma v\rangle$ is the thermally averaged annihilation cross section, and $n_{e q}$ is the number density at thermal equilibrium. For particles of mass $\mathrm{m}$ in the non-relativistic limit, $n_{e q} \propto e^{-m / T}$.

In practice, the density of DM particles falls exponentially with the temperature until DM freezes-out, i.e. until DM falls out of thermal equilibrium. This happens when the annihilation rate $\Gamma=\langle\sigma v\rangle n$ drops below the expansion rate $H$. After freeze-out, the DM density remains constant, and its value is usually expressed as $\Omega=\rho_{D M} / \rho_{c}$, i.e. in units of the critical density $\rho_{c}=3 H^{2} / 8 \pi G$, where $\mathrm{G}$ is the Newton constant.
A useful approximation for the relic density of a DM particle with annihilation cross-section $\langle\sigma v\rangle$ is

$\Omega_{D M}=\frac{3 \times 10^{-27} \mathrm{~cm}^{3} \mathrm{~s}^{-1}}{<\sigma v>}$

The appropriate relic density can thus be achieved with cross sections typical of weak interactions. That's incidentally why one usual refers to DM candidates as WIMPs, fow Weakly Interacting Massive Particles. The calcuation of the actual relic density is usually complicated by particle physics processes including, but not limited to, co-annihilations. We refer the interested reader to the review articles cited above for more information and references.

In this framework, the fact that DM is dark in the local Universe, is due to the fact that it is far too diluted, on average, to produce observable annihilation product. However, this is true only on average, while the theory of structure formation, supported by N-body simulations, predicts the existence of strong inhomogeneities, due to the gravitational growth of density perturbations. Since the annihilation rate depends quadratically on the DM density, it is natural to search for DM annihilation products (photons, neutrinos etc) by looking at regions where DM is expected to accumulate, reaching high densities, thus high annihilation rates, such as the center of galaxies, DM clumps and other targets that will be discussed below.

We stress here that the WIMP paradigm, although appealing and theoretically well motivated, is still tentative, and not conformed by any experimental evidence. DM could be made of particles that never were in thermal equilibrium in the early universe, and in general it could exhibit a phenomenology very different from the one described above. Indirect searches aim precisely at obtaining some insights on the nature of DM, to discriminate WIMP-like scenarios from the many others proposed in literature.

Why gamma-rays? The energy scale of the annihilation products is determined by the mass of the DM particles, as they typically carry a relatively large fraction, say $O(0.1)$, of the available annihilation energy. DM candidates are commonly believed to have masses in the range

$$
20 \mathrm{GeV} \lesssim m_{\chi} \lesssim 120 \mathrm{TeV}
$$

The lower value corresponds to the so-called Lee-Weinberg limit 10,11, valid for fermionic candidates for which the annihilation cross section is proportional to their mass squared $m_{\chi}^{2}$, which has been here updated using the current constraints on the DM relic density. Basically, the lower the mass, the lower $\sigma v$, the higher the relic density. Fermionic thermal relics lighter than $\approx 20 \mathrm{GeV}$ would overclose the Universe, leading to unacceptable values of $\Omega_{D M}$. For realistic, and theoretically well motivated, scenarios, a stronger lower limit comes from null accelerator searches. In the case of Supersymmetric theories, 
the lower limit on the mass of the neutralino (by far the most studied DM candidate), is around $\approx 30 \mathrm{GeV}$, the exact value depending on the specific supersymmetric scenario adopted. Precision electroweak data constrain the inverse compactification scale of Universal Exta Dimension models, thus the mass of the LKP, to be larger than $\approx 300 \mathrm{GeV}[13$.

The upper value comes from the so-called unitarity bound 14 15], which is here re-evaluated by inserting in the old derivation the most recent estimates of the DM relic density.

We stress that both limits are actually model dependent, and should be taken with a grain of salt. Light particles, with $\mathrm{O}(\mathrm{MeV})$ mass, have for instance been proposed as viable DM candidates, evading the Lee-Weinberg limit thanks to the scalar nature of these candidates (see below for more comments and a list of references). Other, very massive, particles could violate the unitarity bound, an example of these candidates are the so-called wimpzillas 16]17.

Why now? Indirect DM searches have been proposed over 25 years ago (an incomplete list of references includes Refs. 18, 19, 20, 21,22,23,24, 25 26]). Since gammaray experiments such as EGRET didn't provide any conclusive evidence for DM particles, why should we trust that present or future telescopes such as CANGAROO [27, GLAST [28], HESS [29, MAGIC [30] or VERITAS 31] will be more succesful? This question is related to another delicate issue: what fraction of the observation time of a gamma-ray telescope should be devoted to DM searches, given the enormous uncertainties associated to the predicted fluxes? The best answer we can give here is that we learned a lot about DM in the last 2 decades, especially on what DM is not. As we argue below, there are indeed good reasons to think that the upcoming generation of gamma-ray, neutrino and antimatter telescopes, with their capability of exploring new windows in the energy spectrum, and already explored windws with unprecedented sensitivity, may eventually find the smoking-gun for DM annihilations, a result that would be of paramount importance for our understanding of the Universe.

\section{Status Quo and Conflicting claims}

The difficulty of obtaining from astrophysical observations conclusive answers on the nature of DM, is witnessed by the numerous conflicting claims of discovery, recently appeared in literature. A number of observations have been in fact "interpreted" in terms of DM, without providing, though, conclusive enough evidence to claim "discovery".

MeV Dark Matter The first example of a "conflicting claim" concerns the so-called Light Dark Matter scenarios. As stressed above, the Lee-Weinberg limit on the mass of the DM particle can be evaded if one e.g. postulates that the DM particle is a scalar. However, there is in principle no reason why one should prefer such a candidate over the more famous, and theoretically well motivated, neutralino. The situation changed, however, after the launch of the INTEGRAL satellite, due to the observation of an intense $511 \mathrm{keV}$ annihilation line from a region of size $\approx 8^{\circ}$ centered around the galactic center 32. This emission did not come as a surprise to astrophysicists, who had discovered the electron-positron annihilation feature already in the early seventies, making it the first extra-solar system spectral feature ever observed 33. The INTEGRAL data however have reopened the old debate on the origin of the positrons annihilating on ambient electrons.

Many astrophysical explanations have been proposed, including production by black holes and pulsars [36], microquasars [37, radioactive nuclei from past supernovae, novae, red giants or Wolf-Rayet stars [35 38, a single recent gamma-ray burst event [39], cosmic ray interactions with the interstellar medium [40] and stellar flares [41. More recently, new scenarios have been proposed invoking pulsar winds 42, primordial and accreting smallmass black holes [43 44] and gamma-ray bursts [45].

The large uncertainties associated with each of these scenarios, however, left the door open to more "exotic" explanations. In particular, the possibility to explain the data in terms of from DM annihilation immediately attracted the attention of particle astrophysicists, also in view of the lack of a disk component of the $511 \mathrm{keV}$ emission, a natural circumstance for a scenario where the positrons would be emitted in a spherically symmetric fashion, following the distribution of DM. However, a simple calculation suggested that any candidate with a mass above the pion mass would inevitably produce gamma-rays and synchrotron emission far above the experimental data. In particular, if the $511 \mathrm{keV}$ emission was due to positrons produced by annihilation of neutralinos, the associated gamma-ray flux would exceed the observed EGRET flux by seven orders of magnitude! A Light DM candidate was instead shown to succesfully reproduce the normalization of the observed $511 \mathrm{keV}$ line without violating any other observational constraint [46]. Following this claim, the interpretation in terms of the annihilation or decay of many other "exotic" candidates have been proposed in literature, including axinos 47, sterile neutrinos 48, scalars with gravitational strength interactions [48, mirror matter [49], color superconducting dark matter [50], superconducting cosmic strings [51], moduli 52 and Q-balls [53].

How to prove that this explanation was right? Clearly, the Light DM intepretation is to be considered tentative until one can find a smoking-gun for it, or make a testable prediction. The first prediction, i.e. the detection of an annihilation line from a dwarf galaxy, has so far failed [54], while further analysises have progressively reduced the allowed parameter space of DM particles. On 
one side, an upper limit on the mass comes from the analysis of Internal Bremsstrahlung emission $(\approx 20 \mathrm{MeV}$, see Ref. [55]) and in-flight annihilation (of order $3-7 \mathrm{MeV}$, see Refs. [56 57]). On the other side, an analysis based on the explosion of the supernova SN1987A sets a lower limit of $\approx 10 \mathrm{MeV}$, thus apparently ruling out Light $\mathrm{DM}$ as a viable explanation of the $511 \mathrm{keV}$ line [58], at least in its most simple realization. Recently, the constraint on Internal-Brehmsstralung has been challenged, and the claim has been made that it is still possible to accomodate all existing constraints while still providing a satisfactory explanation of the INTEGRAL data [59]: the debate is thus still open. Even if all constraints are evaded, however, some fundamental questions remain: how can one prove the "exotic" origin of the positrons, how can one discriminate among different candidates, how to convince a particle physicist that we are dealing with new physics? It appears clear that to promote the Light DM scenario from "tentative intepretation" to "discovery" additional evidence is needed, such as peculiar spectral features (e.g. a $2 \gamma$ line [60]), or discovery in collider searches.

The GeV Excess Another claim of (tentative) discovery has recently been made, based on the analysis of gamma-ray data obtained by EGRET. The evidence in this case would be for WIMPs with a mass of tens of $\mathrm{GeV}$, producing through their annihilation a "bump" in the Galactic gamma-ray emission around $1 \mathrm{GeV}$ 61. Although in principle very exciting, the emission is characterized by a distribution which is very different from the one naïvely predicted by numerical simulations (more intense towards the galactic center), being in the shape of a ring around the galactic center. This is not sufficent of course to rule out this scenario, but there are still numerous difficulties associated with this intepretation, that have been recently highlighted in Ref. 62], in particular regarding the required ring-shaped distribution of $\mathrm{DM}$, as well as the apparent incompatibility with antiproton measurements. As in the case of $\mathrm{MeV} \mathrm{DM}$, this doesn't mean that the proposed interpretation is wrong, but simply that a different approach is needed to obtain conclusive evidence.

Draco Some preliminary results of the CACTUS collaboration [63] have recently been interpreted as a possible evidence for a DM annihilation signal from the Draco dwarf galaxy. Because of the reduced amount of baryons in such a small astrophysical system, an exotic origin of the observed $\mathrm{O}(100) \mathrm{GeV}$ emission was certainly worth being explored. Several authors have investigated this possibility and concluded that the tentative detection was at odds with the expected annihilation signal, as well hard to reconcile with earlier EGRET observations 65. 64. More recently, a re-analysis of the CACTUS data has shown no evidence for an excess of gamma-rays above $100 \mathrm{GeV}$ 66].

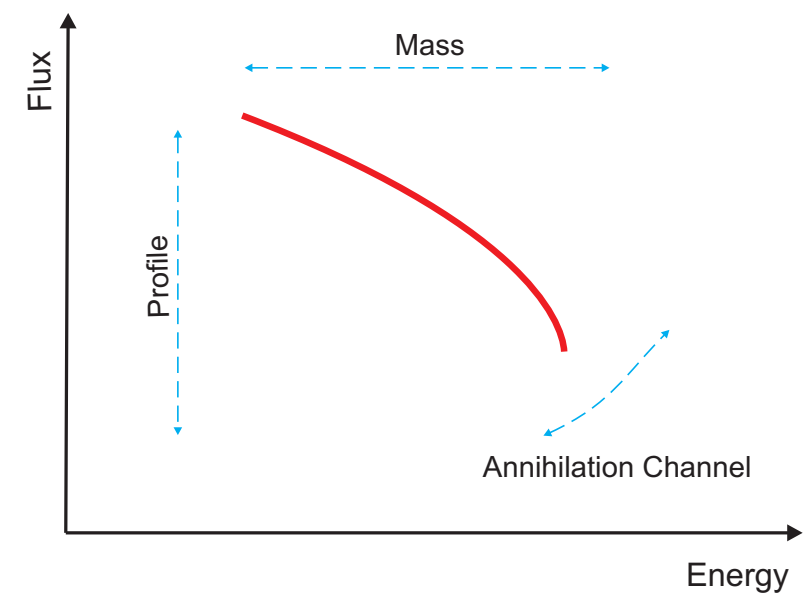

Fig. 1 The problem with indirect searches: the lack of constraints on the mass scale, the profile and the leading annihilation channel, leads to uncertainties on the energy scale and on the spectrum normalization and shape respectively.

The Galactic center The last example of "conflicting claims" is provided by the interpretation of the gammaray source(s) coincident with the Galactic center, in terms of DM annihilation. The discovery of an EGRET source in the direction of $\mathrm{Sgr} \mathrm{A}^{*}$ was in fact a potentially perfect signature of the existence of particle DM, as thoroughly discussed in Refs. 67,68, 69,70,72,73. However, it was subsequently realized that the EGRET source could have been slightly offset with respect to the position of Sgr A*, a circumstance clearly at odds with a DM intepretation [74].

Recently the gamma-ray telescope HESS has detected a high energy source, spatially coincident within $1^{\prime}$ with Sgr A* [75] and with a spectrum extending above $20 \mathrm{TeV}$. Although the spatial coincidence is much more satisfactory than in the case of the EGRET source, the "exotic" origin of the signal is hard to defend, since the implied mass scale of the DM particle (well above $20 \mathrm{TeV}$, to be consistent with the observed spectrum) appears to be difficult to reconcile with the properties of commonly studied candidates, and the fact that the spectrum is a power-law, then, points towards a standard astrophysical source (see e.g. the discussion Ref. [76]). The galactic center, however, remains an interesting target for GLAST, since it will explore a range of energies below the relatively high threshold of HESS, where a DM signal could be hiding [77]. The recent claim that the profile of large galaxies could be much more shallow than previously thought 78, should not discourage further studies, especially in view of the possible enhancement of the DM density due to interactions with the stellar cusp observed at the Galactic center [79.

The detection of a signal from the Galactic center would be extremely interesting, but can it prove the existence of DM? Realistically, one may hope to observe, at most, a "bump" above the background. Without peculiar spectral features it would be hard to claim dis- 


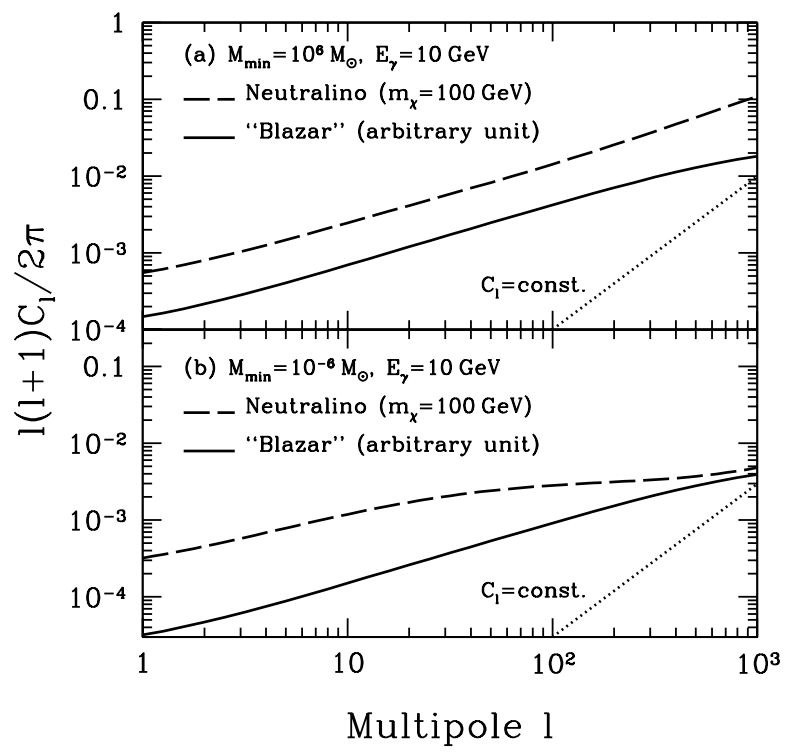

Fig. 2 Shape of the angular power spectrum of the CGB expected from unresolved blazar-like sources (solid lines) with arbitrary normalizations. The power spectrum from annihilation of neutralinos with $m_{\chi}=100 \mathrm{GeV}$ is also plotted as the dashed lines. The adopted gamma-ray energy is $10 \mathrm{GeV}$, and the minimum mass of DM halo is (a) $10^{6} M_{\odot}$, and (b) $10^{-6} M_{\odot}$. The dotted lines show the shot noise $\left(C_{l}=\right.$ const. $)$ with arbitrary normalizations, which represent the power spectrum of very rare sources. From Ref. 83

covery of DM, unless a fit of the spectrum points towards a mass compatible with the eventual findings of new physics searches at accelerators.

Figure 1 illustrates the difficulties associated with the unambiguous identification of a DM signal. Any excess, at any energy, could in principle be explained in terms of DM particles with appropriate properties: the normalization of the flux can be adjusted by changing the distribution of DM particles, the energy scale can be varied over several orders of magnitude, taking advantage of our ignorance on the DM mass scale; even the slope can be modified, since different annihilation channels lead to different spectra.

This doesn't mean that the tentative identifications presented above are ruled-out: the signature of DM could have been already found in one or several sets of data, and all the above claims should be taken seriously and further investigated without prejudice, especially in view of the fact that we don't know what DM is! However, it is important to look for clear smoking-gun of DM annihilation, and study theoretical scenarios with unambiguous signatures that can be tested with present and future experiments. To this aim, we summarize in the next section some recently proposed ideas that go precisely in this direction, and that may shed new light on the nature of particle DM.

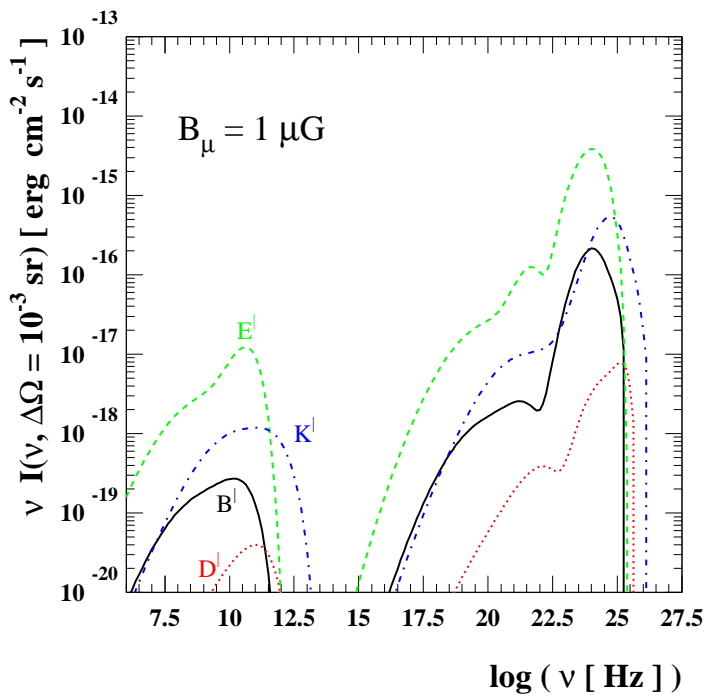

Fig. 3 Multi-wavelength spectra for four different benchmark DM models, for a best fit NFW profile, and a mean magnetic field equal to $1 \mu G$. From Ref. 91, see ibid. for more details.

\section{New strategies}

Before starting the discussion of new strategies for the unambiguous detection of DM, we recall the first, and more clear signature that one may hope to detect: distinctive spectral features, and in particular annihilation lines. This has been discussed thoroughly in literature, and although it appears unlikely that commonly discussed candidates such as the supersymmetric neutralino, possess prominent enough feature to be detected with current or upcoming experiments, it is probably good to keep this possibility in mind, and to search future gamma-data for signatures of this kind.

\subsection{Gamma-ray background}

Although most searches have focused on the identification of point-sources associated with regions where DM accumulates, it is interesting to ask what the gammaray background produced by the annihilations of DM in all structures, at any redshift, would be. The first calculation of this type was performed in Ref. 80], and then further studied in Refs. 81,82. The annihilation background can be expressed as

$\Phi(E)=\frac{\Omega_{D M}^{2} \rho_{c}^{2}}{8 \pi H_{0}} \frac{\sigma v}{m_{\chi}^{2}} \int_{0}^{z_{\max }} \mathrm{d} z \frac{\Delta^{2}}{h(z)} N\left(E^{\prime}\right)$

where $N\left(E^{\prime}\right)$ is the gamma-ray spectrum per annihilation, $H_{0}$ is the Hubble parameter and $h(z)=[(1+$ $\left.z)^{3} \Omega_{D M}+\Omega_{\Lambda}\right]^{1 / 2}$. The information on the shape of individual DM halos in encoded in $\Delta^{2}$, which is essentially the integral of $\rho^{2}$ over the virial volume of the 


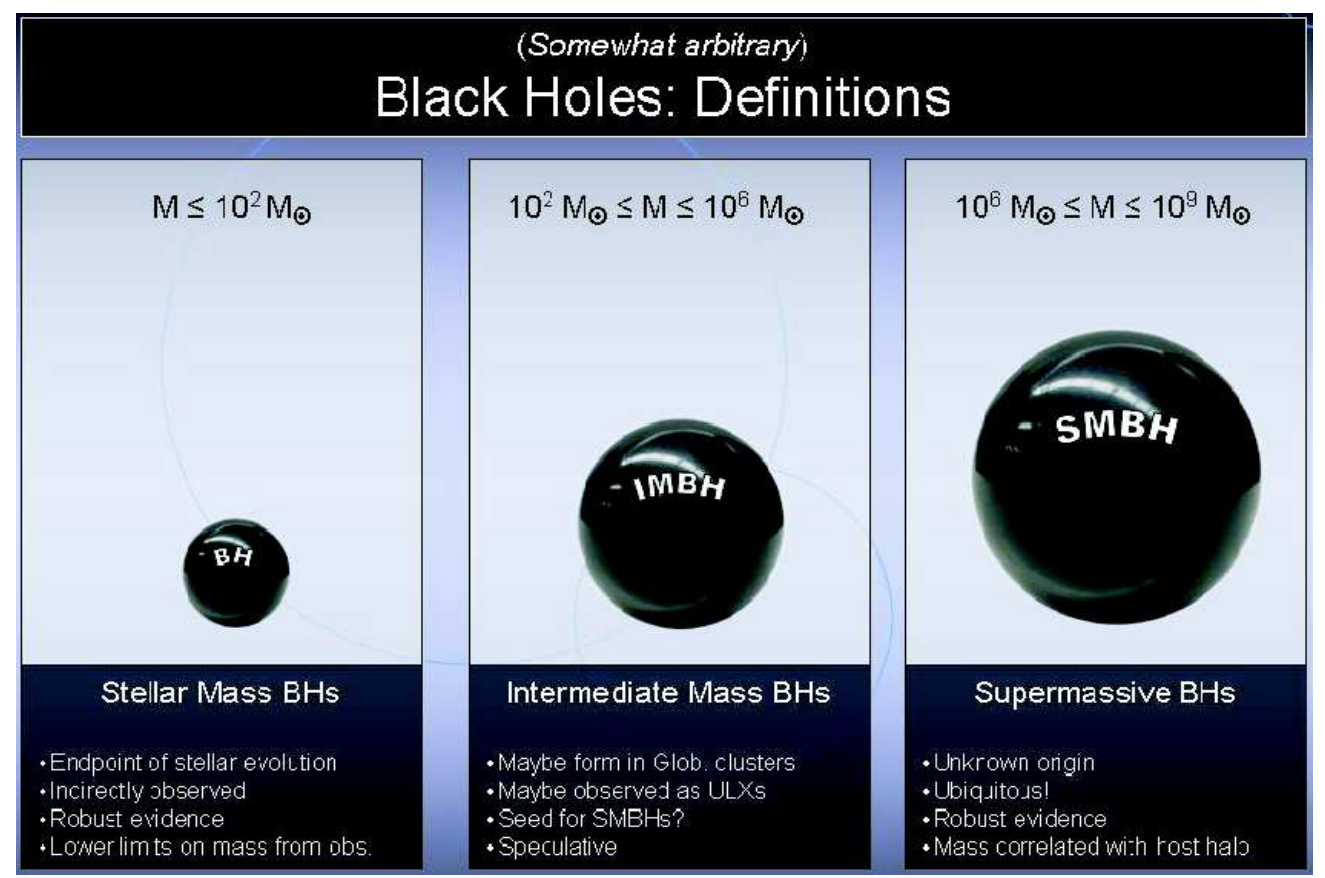

Fig. 4 Classification of Black Holes by mass. There is robust evidence for Stellar mass and Supermassive Black Holes, while Intermediate mass Black Holes are still speculative. IMBHs and SMBHs may act as "annihilation boosters", with important implications for DM searches.

halo. Although it is unlikely that the annihilation background will be detected without first detecting a prominent gamma-ray source at the Galactic center [84, the characteristic power spectrum of the gamma-ray background would discriminate its DM origin from ordinary astrophysical sources 83 . We show in fig. 22 the power spectrum of the gamma-ray background produced by annihilation of neutralinos with $m_{\chi}=100 \mathrm{GeV}$, compared with the one relative to unresolved blazar-like sources. Above $l \sim 200$ the DM spectrum continues to grow whereas the blazar spectrum flattens out, due to the cutoff adopted by the authors corresponding to the minimum mass of halos hosting blazars $\left(\approx 10^{11} M_{\odot}\right)$. The annihilation spectrum thus appear to have much more power at large angular scales, which should be easily distinguished from the blazar spectrum.

There are large uncertainties associated with this calculation, mainly due to our ignorance of the DM profile in the innermost regions of halos, and of the amount of substructures. The existence of mini-spikes (see below) would also dramatically affect the predicted result [85]. But the clear prediction is made that if the observed background has the peculiar shape discussed above, this may be consider as a hint of DM annihilations. Recently the calculation of the neutrino background from DM annihilations has been performed, adopting a formalism very similar to the one sketched above. The comparison with observational data allows to set an interesting, and very general, upper bound on the DM total annihilation cross section 86 .

\subsection{Multi-wavelength}

An alternative strategy is to employ a multi-messenger, multi-wavelength approach. In fact, despite the freedom in the choice of DM parameters makes the intepretation of observational data rather inconclusive, one can always combine the information at different wavelengths, and with different messengers, to obtain more stringent contraints. In fact, gamma-rays are typically (but not exclusively) produced through annihilation and decay chain involving neutral pions

$\chi \bar{\chi} \rightarrow q \bar{q} \rightarrow$ [fragmentation $] \rightarrow \pi^{0} \rightarrow 2 \gamma$

Every time gamma-rays are produced this way, leptons and neutrinos are also produced folloeing the chain

$\chi \bar{\chi} \rightarrow q \bar{q} \rightarrow$ fragmentation $\rightarrow \pi^{ \pm} \rightarrow l, \nu_{l}, \ldots$

An example of this approach is the combined study of the gamma-ray emission from the Galactic center and the associated synchrotron emission produced by the propagation of electron-positron pairs in the Galactic magnetic field 87,71,88,89. Similarly one can investigate what the flux of neutrinos would be, once the gamma-ray flux has been normalized to the EGRET data [90].

One can also ask what the fate of the electron-positron pairs produced by DM annihilation is in dwarf galaxies and clusters of galaxies. An example of this approach can be found in Refs. 91,92, where the authors study the synchrotron and gamma-ray emission from Draco and from the Coma cluster. In fig. 3] we show the multiwavelength spectra of Draco, relative to four different 


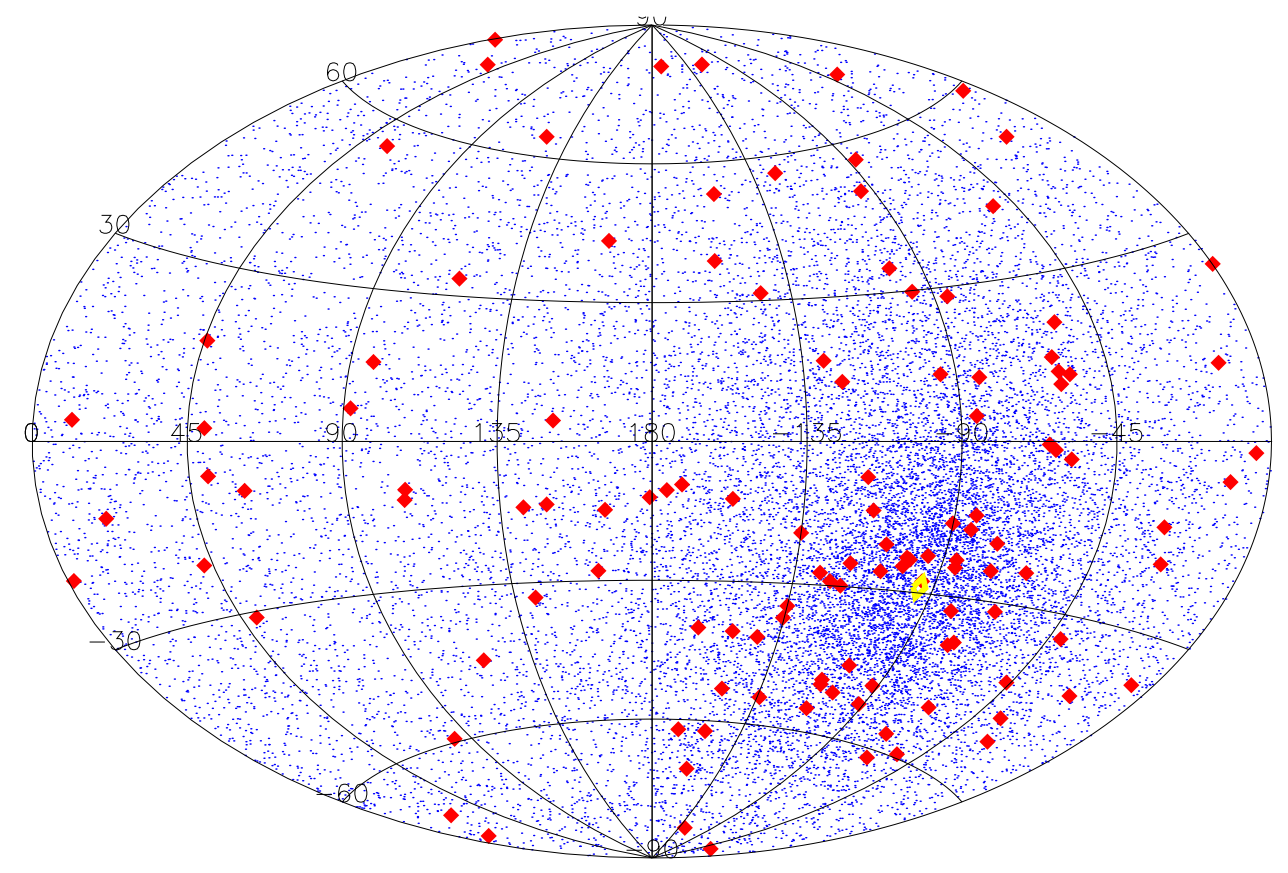

Fig. 5 Sky map in equatorial coordinates showing the position of Intermediate Mass Black Holes in one random realization of a Milky-Way like halo (red diamonds), and in all 200 realizations (blue dots). The concentration at negative declinations corresponds to the position of the Galactic center (black open diamond). From Ref. [11]

DM benchmark models, assuming a NFW profile and a mean magnetic field of $1 \mu G$.

A word of caution is in order, however, when combining information relative to different wavelengths. In fact, not only the available data, due to the different angular resolution of experiments, are relative to different physical regions. But the calculation of the associated spectra at different energies usually requires further inputs, thus introducing new parameters to the problem. The aforementioned calculation of the synchrotron emission is a typical example: Although for every specific DM model, the number of electron-positron pairs produced per annihilation is fixed, the calculation of the synchrotron emission requires an estimate of the diffusion of postrons and it further depends on the magnetic field profile, typically poorly constrained on the scales of interest.

\subsection{Mini-spikes}

Among the new strategies discussed in this section, we will devote particular attention to the effect of black holes (BHs) growth on the surrounding distrbution of DM, a circumstance that will lead us to conclude that BHs can act as DM annihilation "boosters".

Black Holes (BHs) can be broadly divided in 3 different classes, as schematically illustrated in fig. 14 The first class include BHs with mass smaller than $\approx 100$ solar masses, tpypically remnants of the collapse of massive stars (recent simulations suggest that the upper limit on the mass of these objects is as low as $\approx 20 M_{\odot} \quad$ 93]). There is robust evidence for the existence of these objects, coming from the observation of binary objects with compact objects whose mass exceeds the critical mass of Neutron Stars. For a review of the topic and the discussion of the possible smoking-gun for Stellar Mass BHs see e.g. 94 and references therein.

The existence of Supermassive BHs (SMBHs), lying at the center of galaxies (including our own), is also well-established (see e.g. Ref. 95]), and intriguing correlations are observed between the BHs mass and the properties of their host galaxies and halos $96,97,98,99$, 100 110. From a theoretical point of view, a population of massive seed black holes could help to explain the origin of SMBHs. In fact, observations of quasars at redshift $z \approx 6$ in the Sloan Digital survey [101,102 103 suggest that SMBHs were already in place when the Universe was only $\sim 1$ Gyr old, a circumstance that can be understood in terms of rapid growth starting from "massive" seeds (see e.g. Ref. [104).

This leads us to the third category of BHs, characterized by their intermediate mass. In fact, scenarios that seek to explain the properties of the observed supermassive black holes population result in the prediction of a large population of wandering Intermediate Mass BHs (IMBHs). Here, following Ref. [105, we consider two different formation scenarios for IMBHs. In the first scenario, IMBHs form in rare, overdense regions at high 
redshift, $z \sim 20$, as remnants of Population III stars, and have a characteristic mass-scale of a few $10^{2} M_{\odot}$ [106 (a similar scenario was investigated in Ref. 107 108 109]). In this scenario, these black holes serve as the seeds for the growth supermassive black holes found in galactic spheriods 95. In the second scenario, IMBHs form directly out of cold gas in early-forming halos and and are typified by a larger mass scale, of order $10^{5} M_{\odot}$ [110. In Fig. 5 we show the distribution of IMBHs in the latter scenario, as obtained in Ref. 111.

We have so far discussed about BHs, but we haven't yet established the connection with DM searches. The effect of the formation of a central object on the surrounding distribution of matter has been investigated in Refs. 112,113, 114, 115 and for the first time in the framework of DM annihilations in Ref. 116. It was shown that the adiabatic growth of a massive object at the center of a power-law distribution of DM with index $\gamma$, induces a redistribution of matter into a new power-law (dubbed "spike") with index $\gamma_{s p}=(9-2 \gamma) /(4-\gamma)$ This formula is valid over a region of size $R_{s} \approx 0.2 r_{B H}$, where $r_{B H}$ is the radius of gravitational influence of the black hole, defined implicitly as $M\left(<r_{B H}\right)=M_{B H}$, with $M(<r)$ mass of the DM distribution within a sphere of radius $r$, and $M_{B H}$ mass of the Black Hole [117. The process adiabatic growth is in particular valid for the SMBH at the Galactic center. A critical assessment of the formation and survival of the central spike, over cosmological timescales, is presented in Refs. 119 118. (see also references therein).

Here we will not further discuss the spike at the Galactic center, and will rather focus our attention on mini-spikes around IMBHs. If $N_{\gamma}(E)$ is the spectrum of gamma-rays per annihilation, the gamma-ray flux from an individual mini-spike can be expressed as 105]

$\Phi_{\gamma}(E)=\phi_{0} m_{\chi, 100}^{-2}(\sigma v)_{26} D_{\mathrm{kpc}}^{-2} L_{\mathrm{sp}} N_{\gamma}(E)$

with $\phi_{0}=9 \times 10^{-10} \mathrm{~cm}^{-2} \mathrm{~s}^{-1}$. The first two factors depend on the particle physics parameters, viz. the mass of the DM particle in units of $100 \mathrm{GeV} m_{\chi, 100}$, and its annihilation cross section in units of $10^{-26} \mathrm{~cm}^{3} / \mathrm{s},(\sigma v)_{26}$, while the third factor accounts for the flux dilution with the square of the IMBH distance to the Earth in kpc, $D_{\mathrm{kpc}}$. Finally, the normalization of the flux is fixed by an adimensional luminosity factor $L_{\mathrm{sp}}$, that depends on the specific properties of individual spikes. In the case where the DM profile before the formation of the IMBH follows the commonly adopted Navarro, Frenk and White profile [120, the final DM density $\rho(r)$ around the IMBH will be described by a power law $r^{-7 / 3}$ in a region of size $R_{s}$ around the IMBHs. Annihilations themselves will set an upper limit to the DM density $\rho_{\max } \approx m_{\chi} /[(\sigma v) t]$, where $t$ is the time elapsed since the formation of the minispike, and we denote with $R_{\mathrm{c}}$ the "cut" radius where $\rho\left(R_{\mathrm{c}}\right)=\rho_{\max }$. With these definitions, the intrinsic luminosity factor in Eq. [6] reads

$L_{\mathrm{sp}} \equiv \rho_{100}^{2}\left(R_{\mathrm{s}}\right) R_{\mathrm{s}, \mathrm{pc}}^{14 / 3} R_{\mathrm{c}, \mathrm{mpc}}^{-5 / 3}$ where $R_{\mathrm{s}, \mathrm{pc}}$ and $R_{\mathrm{c}, \mathrm{mpc}}$ denote respectively $R_{\mathrm{s}}$ in parsecs and $R_{\mathrm{c}}$ in units of $10^{-3} \mathrm{pc}, \rho_{100}(r)$ is the density in units of $100 \mathrm{GeV} \mathrm{cm}^{-3}$. Typical values of $L_{\mathrm{sp}}$ lie in the range $0.1-10$ [105].

In Fig. [6] we show the (average) integrated luminosity function of IMBHs in scenario B. We define the integrated luminosity function as the number of black holes producing a gamma-ray flux larger than $\Phi$, as a function of $\Phi$. Loosely speaking, this can be understood as he number of mini-spikes that can be detected with an experiment with point source sensitivity $\Phi$ above $1 \mathrm{GeV}$. The upper (lower) line corresponds to $m_{\chi}=100 \mathrm{GeV}$, $\sigma v=3 \times 10^{-26} \mathrm{~cm}^{3} \mathrm{~s}^{-1}\left(m_{\chi}=1 \mathrm{TeV}, \sigma v=10^{-29} \mathrm{~cm}^{3} \mathrm{~s}^{-1}\right)$. We show for comparison the point source sensitivity above $1 \mathrm{GeV}$ for EGRET and GLAST, corresponding roughly to the flux for a $5 \sigma$ detection of a high-latitude pointsource in an observation time of 1 year [121. The dashed region corresponds to the $1 \sigma$ scatter between different realizations of Milky Way-sized halos. This band includes the variation in spatial distributions of IMBHs from one halo to the next as well as the variation in the individual properties of each IMBH in each realization. GLAST may thus be able to detect up to 100 point sources with identical spectra, and not correlated with the disk, thus providing compelling evidence for the non-astrophysical origin of their gamma-ray emission. If DM is heavy, above $\approx 300 \mathrm{GeV}$, Air Cherenkov Telescopes can be used to extend the observation of GLAST sources to higher energies. At the same time, the annihilation of heavy particles to neutrinos may lead to interesting signatures in neutrino telescopes 111.

\section{Conclusions}

To establish the connection between gamma-ray astrophysics and DM searches, we have first reviewed the fundamental motivations for indirect DM searches. We tried to convey the fundamental message, especially to non-experts, that despite the "exotic" nature of the particles under consideration, indirect signals of DM annihilation are a "natural" expectation in the standard WIMP paradigm, and that it is certainly worth pursuing further indirect DM searches. We have also provided a critical assessment of some recent "conflicting claims" of discovery, recently appeared in literature. Without taking sides in the disputes over specific DM candidates, we have stressed the difficulty of making unambiguous claims, especially in view of the large uncertainties associated with the mass scale, the distribution and the nature of DM. We have finally shown that despite these conflicting claims, the "status quo" shouldn't discourage further studies, especially in view of the numerous new strategies that have been recently proposed, and that may provide unambiguous evidence for DM. Among them we have discussed the peculiar power-spectrum of the gamma-ray background from DM annihilations; the 


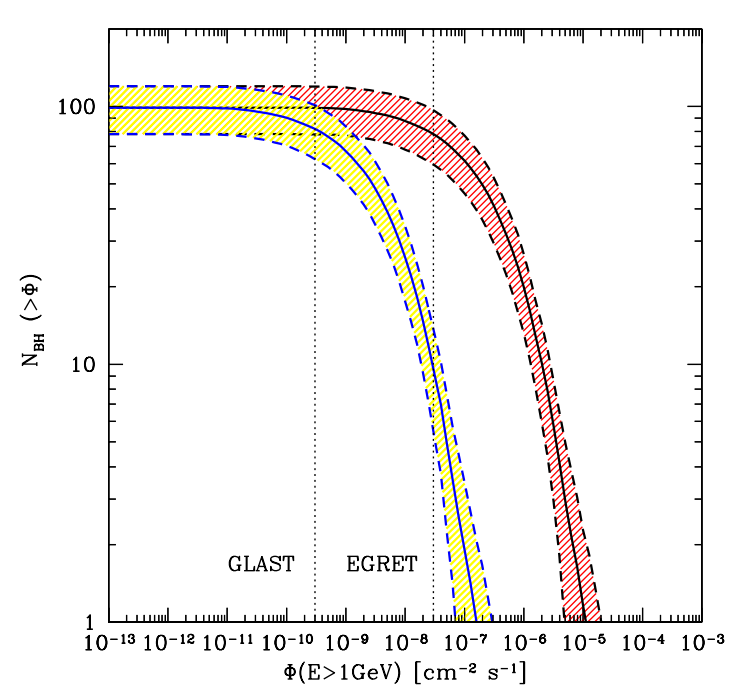

Fig. 6 IMBHs integrated luminosity function, (number of mini-spikes detectable with an experiment of sensitivity $\phi$ ) for IMBHs with mass $\sim 10^{5} M_{\odot}$. The upper (lower) line corresponds to $m_{\chi}=100 \mathrm{GeV}, \sigma v=3 \times 10^{-26} \mathrm{~cm}^{3} \mathrm{~s}^{-1}\left(m_{\chi}=1\right.$ $\mathrm{TeV}, \sigma v=10^{-29} \mathrm{~cm}^{3} \mathrm{~s}^{-1}$ ). For each curve we also show the $1-\sigma$ scatter among different realizations of Milky Way-sized host DM halos. We show for comparison the $5 \sigma$ point source sensitivity above $1 \mathrm{GeV}$ of EGRET and GLAST (1 year). From Ref. 105.

multi-wavelength, multi-messenger approach; and the socalled "mini-spike" scenario, where IMBHs act as "boosters" of the DM annihilation signal.

Acknowledgements I thank my collaborators J. Beacom, N. Bell, D. Merritt, E. Nezri, J. Orloff, G. Servant, G. Sigl, J. Silk and A. Zentner. It is a pleasure to thank the organizers of the Barcelona Workshop for a stimulating and well organized conference. This work was supported by the Helmholtz Association of National Research Centres, project VH-NG-006.

\section{References}

1. L. Bergstrom, Rept. Prog. Phys. 63 (2000) 793 arXiv:hep-ph/0002126.

2. G. Bertone, D. Hooper and J. Silk, Phys. Rept. 405 (2005) 279

3. D. Hooper and A. M. Taylor, arXiv:hep-ph/0607086

4. D. R. Tovey, Eur. Phys. J. directC 4 (2002) N4.

5. E. A. Baltz, M. Battaglia, M. E. Peskin and T. Wizansky, arXiv:hep-ph/0602187

6. G. Servant and T. M. P. Tait, Nucl. Phys. B 650 (2003) 391 arXiv:hep-ph/0206071.

7. T. Appelquist, H. C. Cheng and B. A. Dobrescu, Phys. Rev. D 64 (2001) 035002 arXiv:hep-ph/0012100.

8. A. Datta, K. Kong and K. T. Matchev, Phys. Rev. D 72 (2005) 096006 [Erratum-ibid. D $\mathbf{7 2}$ (2005) 119901] arXiv:hep-ph/0509246.

9. E. W. Kolb and M. S. Turner, "The Early Universe".

10. B. W. Lee and S. Weinberg, Phys. Rev. Lett. 39, 165 (1977).
11. P. Hut, Phys. Lett. B 69 (1977) 85.

12. S. Eidelman et al. [Particle Data Group], Phys. Lett. B 592 (2004) 1.

13. I. Gogoladze and C. Macesanu, arXiv:hep-ph/0605207

14. S. Weinberg, 1995, The Quantum Theory of Fields Vol 1: Foundations, Cambridge University Press.

15. K. Griest and M. Kamionkowski, Phys. Rev. Lett. 64 (1990) 615.

16. E. W. Kolb, D. J. Chung and A. Riotto, Proceedings of the 2nd International Conference on Dark Matter in Astro and Particle Physics (DARK98), Heidelberg, Germany, (1998) arXiv:hep-ph/9810361.

17. S. Chang, C. Coriano and A. E. Faraggi, Nucl. Phys. B 477, 65 (1996) arXiv:hep-ph/9605325.

18. J. E. Gunn, B. W. Lee, 1. Lerche, D. N. Schramm and G. Steigman, Astrophys. J. 223, 1015 (1978).

19. F. W. Stecker, Astrophys. J. 223, 1032 (1978).

20. M. Srednicki, S. Theisen and J. Silk, Phys. Rev. Lett. 56, 263 (1986) [Erratum-ibid. 56, 1883 (1986)].

21. S. Rudaz, Phys. Rev. Lett. 56, 2128 (1986).

22. L. Bergstrom and H. Snellman, Phys. Rev. D 37, 3737 (1988).

23. F. W. Stecker, Phys. Lett. B 201, 529 (1988).

24. F. W. Stecker and A. J. Tylka, Astrophys. J. 343, 169 (1989).

25. A. Bouquet, P. Salati and J. Silk, Phys. Rev. D 40, 3168 (1989).

26. S. Rudaz and F. W. Stecker, Astrophys. J. 368, 406 (1991).

27. http://icrhp9.icrr.u-tokyo.ac.jp/index.html

28. http://www-glast.stantord.edu/

29. http://www.mpl-hd.mpg.de/htm/HESS/HESS.html

30. http://hegral.mppmu.mpg.de/MAGICWeb/

31. http://veritas.sao.arizona.edu/index.html

32. J. Knodlseder et al., Astron. Astrophys. 441 (2005) 513 arXiv:astro-ph/0506026.

33. Johnson, W. N., III, \& Haymes, R. C. Astrophys. J. 184, $103(1973)$

34. C. D. Dermer and R. J. Murphy, Proc. Fourth INTEGRAL Workshop. ESA SP-459, ISBN 92-9092-677-5, 115. arXiv:astro-ph/0107216.

35. M. Cassé, B. Cordier, J. Paul and S. Schanne, Astrophys. J. 602, L17 (2004) arXiv:astro-ph/0309824.

36. C. D. Dermer and E. P. Liang, Nuclear Spectroscopy of Astrophysical Sources, ed. N. Gehrels and G. H. Share (New York: AIP), 326.

37. H. Li and E. P. Liang, Astrophys. J. 458, 514 (1996) arXiv:astro-ph/9509024.

38. M. Signore and G. Vedrenne, Astron. Astrophys. 201, 379 (1988).

39. S. R. Furlanetto and A. Loeb, Astrophys. J. 569, L91 (2002) arXiv:astro-ph/0203044.

40. B. Kozlowsky, R. E. Lingenfelter and R. Ramaty, Astrophys. J. 316, 801 (1987).

41. G. Meynet, M. Arnould, N. Prantzos and G. Paulus, Astron. Astrophys. 320, 460 (1997).

42. W. Wang, C. S. J. Pun and K. S. Cheng, arXiv:astro-ph/0509760

43. P. H. Frampton and 'T. W. Kephart, Mod. Phys. Lett. A 20, 1573 (2005) arXiv:hep-ph/0503267.

44. L. Titarchuk and P. Chardonnet, arXiv:astro-ph/0511333

45. G. Bertone, A. Kusenko, S. Palomares-Ruiz, S. Pascoli and D. Semikoz, Phys. Lett. B 636 (2006) 20 arXiv:astro-ph/0405005.

46. C. Boehm, D. Hooper, J. Silk, M. Casse and J. Paul, Phys. Rev. Lett. 92 (2004) 101301 arXiv:astro-ph/0309686 .

47. D. Hooper and L. T. Wang, Phys. Rev. D 70, 063506 (2004) arXiv:hep-ph/0402220. 
48. C. Picciotto and M. Pospelov, Phys. Lett. B 605, 15 (2005) arXiv:hep-ph/0402178.

49. R. Foot and Z. K. Silagadze, Int. J. Mod. Phys. D 14, 143 (2005) arXiv:astro-ph/0404515.

50. D. H. Oaknin and A. R. Zhitnitsky, Phys. Rev. Lett. 94, 101301 (2005) arXiv:hep-ph/0406146.

51. F. Ferrer and T. Vachaspatı, arXıv:astro-ph/0505063

52. M. Kawasaki and T. Yanagida, Phys. Lett. B 624, 162 (2005) arXiv:hep-ph/0505167.

53. S. Kasuya and F'. lakahashı, Phys. Rev. D 72, 085015 (2005) arXiv:astro-ph/0508391.

54. B. Cordier et al., arXıv:astro-ph/0404499

55. J. F. Beacom, N. F. Bell and G. Bertone, Phys. Rev. Lett. 94, 171301 (2005) arXiv:astro-ph/0409403.

56. J. F. Beacom and H. Yuksel, Phys. Rev. Lett. 97, 071102 (2006) arXiv:astro-ph/0512411.

57. P. Sizun, M. Casse and S. Schanne, arXiv:astro-ph/0607374

58. P. Fayet, D. Hooper and G. Sigl, Phys. Rev. Lett. 96, 211302 (2006) arXiv:hep-ph/0602169.

59. C. Boehm and P. Uwer, arXiv:hep-ph/0606058

60. C. Boehm, J. Orlott and P. Salati, arXiv:astro-ph/0607437

61. W. de Boer, C. Sander, V. Zhukov, A. V. Gladyshev and D. I. Kazakov, Phys. Rev. Lett. 95 (2005) 209001 arXiv:astro-ph/0602325.

62. L. Bergstrom, J. Edsjo, M. Gustafsson and P. Salati, JCAP 0605 (2006) 006 arXiv:astro-ph/0602632.

63. P. Marleau, TAUP, Zaragoza, Spain, September 2005; M. Tripathi, Cosmic Rays to Colliders 2005, Prague, Czech Republic, September 2005; TeV Particle Astrophysics Workshop, Batavia, USA, July 2005; M. Chertok, PANIC 05, Santa Fe, USA, October 2005.

64. S. Profumo and M. Kamionkowski, JCAP 0603 (2006) 003 arXiv:astro-ph/0601249.

65. L. Bergstrom and D. Hooper, Phys. Rev. D 73 (2006) 063510 arXiv:hep-ph/0512317.

66. M. Tripathı, private communication

67. F. W. Stecker, Phys. Lett. B 201, 529 (1988)

68. A. Bouquet, P. Salati, and J. Silk, Phys. Rev. D 40, 3168 (1989).

69. V. Berezinsky, A. Bottino, and G. Mignola, Phys. Lett. $B$ 325, 136 (1994)

70. L. Bergstrom, P. Ullio and J. H. Buckley, Astropart. Phys. 9, 137 (1998)

71. G. Bertone, G. Sigl and J. Silk, Mon. Not. Roy. Astron. Soc. 326 (2001) 799 arXiv:astro-ph/0101134.

72. A. Cesarini, F. Fucito, A. Lionetto, A. Morselli and P. Ullio, Astropart. Phys. 21, 267 (2004) arXiv:astro-ph/0305075.

73. N. Fornengo, L. Pierı and S. Scopel, Phys. Rev. D 70 (2004) 103529 arXiv:hep-ph/0407342.

74. D. Hooper and B. L. Dingus, Phys. Rev. D 70 (2004) 113007 arXiv:astro-ph/0210617

75. F. Aharonian et al. [The HESS Collaboration], Astron. Astrophys. 425 (2004) L13 arXiv:astro-ph/0408145.

76. S. Profumo, Phys. Rev. D 72, 103521 (2005) arXiv:astro-ph/0508628

77. G. Zaharilas and D. Hooper, Phys. Rev. D 73, 103501 (2006) arXiv:astro-ph/0603540.

78. S. Mashchenko, H. M. P. Couchman and J. Wadsley, Nature 442 (2006) 539 arXiv:astro-ph/0605672.

79. D. Merritt, S. Harfst and G. Bertone, in preparation

80. L. Bergstrom, J. Edsio and P. Ullio, Phys. Rev. Lett. 87 (2001) 251301 arXiv:astro-ph/0105048.

81. J. E. Taylor and J. Silk, Mon. Not. Roy. Astron. Soc. 339 (2003) 505 arXiv:astro-ph/0207299.

82. P. Ullio, L. Bergstrom, J. Edsjo and C. Lacey, Phys. Rev. D 66 (2002) 123502 arXiv:astro-ph/0207125.

83. S. Ando and E. Komatsu, Phys. Kev. D 73 (2006) 023521 arXiv:astro-ph/0512217.
84. S. Ando, arXiv:astro-ph/0503006

85. S. Horiuchl and S. Ando, arXıv:astro-ph/0607042

86. J. F. Beacom, N. F. Bell and G. D. Mack, arXiv:astro-ph/0608090

87. P. Gondolo, Phys. Lett. B 494, 181 (2000).

88. G. Bertone, G. Servant and G. Sigl, Phys. Rev. D 68 (2003) 044008 arXiv:hep-ph/0211342

89. R. Aloisio, P. Blası and A. V. Ulinto, JCAP 0405, 007 (2004) arXiv:astro-ph/0402588.

90. G. Bertone, E. Nezrı, J. Urlott and J. Silk, Phys. Rev. D 70, 063503 (2004) arXiv:astro-ph/0403322.

91. S. Colafrancesco, S. Protumo and P. Ullio, arXiv:astro-ph/0607073

92. S. Colatrancesco, S. Profumo and P. Ullio, arXiv:astro-ph/0507575

93. Fryer, C. L., \& Kalogera, V. 2001, Astrophys. J. 554, 548

94. R. Narayan, arXiv:astro-ph/0310692

95. Ferrarese, L., \& Ford, H. 2005, Space Science Reviews, 116,523

96. Kormendy, J., \& Richstone, D. 1995, Ann. Rev. Astron. \& Astrophys., 33, 581

97. L. Ferrarese and D. Merritt, Astrophys. J. 539 (2000) L9 arXiv:astro-ph/0006053.

98. R. J. McLure and J. S. Dunlop, Mon. Not. Roy. Astron. Soc. 331 (2002) 795 arXiv:astro-ph/0108417.

99. K. Gebhardt et al., Astrophys. J. 539 (2000) L13 arXiv:astro-ph/0006289.

100. S. Tremaine et al., Astrophys. J. 574 (2002) 740 arXiv:astro-ph/0203468.

101. X. Fan et al. [SDSS Collaboration], Astron. J. 122 (2001) 2833 arXiv:astro-ph/0108063.

102. Barth, A. J., Martini, P., Nelson, C. H., \& Ho, L. C. 2003, Astrophys. Lett. 594, L95

103. C. J. Willott, R. J. McLure and M. J. Jarvis, Astrophys. J. 587 (2003) L15 arXiv:astro-ph/0303062

104. Haiman, Z., \& Loeb, A. 2001, Astrophys. J. , 552, 459

105. G. Bertone, A. R. Zentner and J. Silk, Phys. Rev. D 72 (2005) 103517 arXiv:astro-ph/0509565.

106. Madau, P., \& Rees, M. J. 2001, Astrophys. J. Lett. 551, L27

107. H. S. Zhao and J. Silk, arXiv:astro-ph/0501625

108. R. Islam, J. Taylor and J. Silk, Mon. Not. Roy. Astron. Soc. 354 (2003) 443

109. R. Islam, J. Taylor and J. Silk, Mon. Not. Roy. Astron. Soc. 354 (2004) 427

110. S. M. Koushiappas, J. S. Bullock and A. Dekel, Mon. Not. Roy. Astron. Soc. 354 (2004) 292 arXiv:astro-ph/0311487.

111. G. Bertone, Phys. Rev. D 73 (2006) 103519 arXiv:astro-ph/0603148.

112. Peebles, P. J. E. 1972, Astrophys. J. 178, 371

113. P. Young, 1980, Astrophys. J. 242 (1980), 1232

114. J. R. Ipser and P. Sikivie, Phys. Rev. D 35 (1987) 3695.

115. Quinlan, G. D., Hernquist, L., \& Sigurdsson, S. 1995, Astrophys. J. 440, 554

116. P. Gondolo and J. Silk, Phys. Rev. Lett. 83 (1999) 1719 arXiv:astro-ph/9906391.

117. D. Merritt, Proceedings of Carnegie Observatories Centennial Symposium Coevolution of Black Holes and Galaxies arXiv:astro-ph/0301257.

118. G. Bertone and D. Merritt, Mod. Phys. Lett. A 20 (2005) 1021 arXiv:astro-ph/0504422.

119. G. Bertone and D. Merritt, Phys. Rev. D 72 (2005) 103502 arXiv:astro-ph/0501555.

120. J. F. Navarro, C. S. Frenk and S. D. M. White, Astrophys. J. 490 (1997) 493.

121. A. Morselli, A. Lionetto, A. Cesarini, F. Fucito and P. Ullio [GLAST Collaboration], Nucl. Phys. Proc. Suppl. 113 (2002) 213 arXiv:astro-ph/0211327. 